\title{
Characterization of Graphs Associated with the Ideal of Numerical Semigroups
}

\author{
Peng Xu, ${ }^{1,2}$ Muhammad Ahsan Binyamin (D), ${ }^{3}$ Adnan Aslam $\left(\mathbb{D},{ }^{4}\right.$ Wajid Ali, ${ }^{3}$ \\ Hasan Mahmood, ${ }^{5}$ and Hao Zhou ${ }^{6}$ \\ ${ }^{1}$ Institute of Computational Science and Technology, Guangzhou University, Guangzhou, China \\ ${ }^{2}$ School of Computer Science of Information Technology, Qiannan Normal University for Nationalities, Duyun 558000, China \\ ${ }^{3}$ Department of Mathematics, GC University, Faisalabad, Pakistan \\ ${ }^{4}$ Department of Natural Sciences and Humanities, University of Engineering and Technology, Lahore, Pakistan \\ ${ }^{5}$ Department of Mathematics, GC University, Lahore, Pakistan \\ ${ }^{6}$ Transportation School of Wuhan University of Technology, Wuhan University of Technology, Wuhan 430070, China
}

Correspondence should be addressed to Muhammad Ahsan Binyamin; ahsanbanyamin@gmail.com

Received 29 June 2020; Accepted 30 July 2020; Published 7 September 2020

Academic Editor: Marco Fontana

Copyright (c 2020 Peng Xu et al. This is an open access article distributed under the Creative Commons Attribution License, which permits unrestricted use, distribution, and reproduction in any medium, provided the original work is properly cited.

Let $I$ be an ideal of a numerical semigroup $\Lambda$. We define an undirected graph $G_{I}(\Lambda)$ with vertex set $\left\{v_{i}: i \in(\Lambda \backslash I)^{*}=(\Lambda \backslash I)-\{0\}\right\}$ and edge set $\left\{v_{i} v_{j} \Longleftrightarrow i+j \in I\right\}$. The aim of this article is to discuss the connectedness, girth, completeness, and some other related properties of the graph $G_{I}(\Lambda)$.

\section{Introduction and Preliminaries}

In mathematics, graph theory plays an important role in understanding the relationship between pair of objects through graphs. Today, graph theory has a number of applications in the field of engineering, computer science, and other sciences (see [1-3]). The idea of linking graph with algebraic structure gave rise to new research in the field of mathematics. This idea explores the interaction between structures of algebraic objects and graphs by using properties of graphs and algebraic structures. In the past, many researchers associated graphs with different algebraic structures (see [4-11]).

A graph $G(V(G), E(G))$ is an ordered pair with the vertex set $V(G)$ and the edge set $E(G)$. The order and the size of the graph $G$ are the cardinality of its vertex set and edge, respectively. A graph $G$ is connected if for any two vertices $u, v \in V(G)$, there is a path connecting $u$ and $v$. The distance between two vertices $u, v \in V(G)$ is the length of the shortest path between them and is denoted by $d(u, v)$. The length of the longest path in $G$ is the diameter of $G$, denoted by $\operatorname{diam}(G)$. Length of a shortest cycle in the graph is referred to as the girth of the graph. A graph is complete if for any two vertices, there is an edge between them. A subgraph $H$ of a graph $G$ is a clique if $H$ is a complete graph, and the order of maximal clique is called the clique number of $G$, denoted by $c l(G)$. A graph is regular if all of its vertices have the same degree. For more undefined terminologies related to graph theory, see [12-14].

Numerical semigroup theory is the study of set of positive integer solutions of diophantine equations (see [15-18]), and this is very useful in the study of algebraic geometry codes (see [19-21]). Let us recall some definitions from the numerical semigroup theory, which we used in the later sections.

A subset $\Lambda \subseteq \mathbb{N}$ of nonnegative integers is called submonoid if the following holds:

(1) $0 \in \Lambda$

(2) If $x, y \in \Lambda$, then $x+y \in \Lambda$

A submonoid $\Lambda$ is called numerical semigroup if $\mathbb{N} \backslash \Lambda$ is finite. The least positive integer in $\Lambda$, denoted by $m_{\Lambda}$, is known as the multiplicity of the numerical semigroup. The 
elements of $\mathbb{N} \backslash \Lambda$ are called the gaps of $\Lambda$, and the largest of these gaps is known as the Frobenius number, denoted by $F_{\Lambda}$. It is well known that every numerical semigroup is finitely generated; that is, there exist $a_{1}, \ldots, a_{t}$ such that $\Lambda=\left\langle a_{1}, \ldots, a_{t}\right\rangle=\left\{n_{1} a_{1}+\cdots n_{t} a_{t}: n_{1}, \ldots, n_{t} \in \mathbb{N}\right\}$. Moreover, every numerical semigroup has a unique minimal system of generators. The cardinality of the minimal system of generators is called the embedding dimension of numerical semigroup $\Lambda$, denoted by $e_{\Lambda}$. A subset $I$ of numerical semigroup $\Lambda$ is ideal (integral ideal) of $\Lambda$ if $I+\Lambda \subseteq I$ (that is, $\forall x \in I$ and $s \in \Lambda$, the element $x+s$ is in $I$ ). An ideal $I$ is an irreducible ideal if it cannot be written as intersection of two ideals which properly contain it.

A connection of graphs with numerical semigroups can be found in [22]. In this paper, we investigated the interplay between the structure of numerical semigroups and graph theoretic properties of specific graphs gained from numerical semigroups. The sketch of this paper is as follows.

In Section 2, we briefly describe the concept of connectedness, diameter, and girth of $G_{I}(\Lambda)$. In Section 3, we present a necessary and sufficient condition for a graph associated with an ideal of a numerical semigroup to be complete. In Section 4, we discuss the concept of clique number and cut point of $G_{I}(\Lambda)$.

\section{Connectedness, Diameter, and Girth of $G_{I}(\Lambda)$}

We start this section by showing that $G_{I}(\Lambda)$ is always a connected graph. Also, we show that the diameter of $G_{I}(\Lambda)$ is equal or less than 2 and girth of $G_{I}(\Lambda)$ is 3 .

Proposition 1. Let $\Lambda$ be a numerical semigroup and I be an ideal of $\Lambda$. Then, $G_{I}(\Lambda)$ is a connected graph of finite order with at least one vertex of degree $\left|(\Lambda \backslash I)^{*}\right|-1$.

Proof. Choose $F_{\Lambda} \in \Lambda$, the Frobenius number, and $l \in I$, the least element. Then, note that $\left(F_{\Lambda}+l\right)+m \in I$ for all $m \geq 1$. This gives that $\Lambda \backslash I$ is always finite, and therefore, $(\Lambda \backslash I)^{*}$ is finite. We may assume that $n$ is the largest element of $\Lambda$ not in $I$ as $(\Lambda \backslash I)^{*}$ is finite. This gives that $i+n \in I, \forall i \in(\Lambda \backslash I)^{*}$. Therefore, $G_{I}(\Lambda)$ is a connected graph of finite order with degree of $v_{n}$ and is equal to $\left|(\Lambda \backslash I)^{*}\right|-1$.

Example 1. Let $\Lambda=\{0,3,6,7,9,10,11,12,13,14, \ldots\}$ be a numerical semigroup and $I=\{11,12,13, \ldots\}$ be an ideal of $\Lambda$. Then, $(\Lambda \backslash I)^{*}=\{3,6,7,9,10\}$ and the graph $G_{I}(\Lambda)$ is shown in Figure 1.

Proposition 2. Let $G_{I}(\Lambda)$ be a graph associated with an ideal I of a numerical semigroup $\Lambda$. Then, $\operatorname{diam}\left(G_{I}(\Lambda)\right) \leq 2$. Furthermore, if $G_{I}(\Lambda)$ contains a cycle, then $\operatorname{gr}\left(G_{I}(\Lambda)\right) \leq 5$.

Proof. Let $n \in(\Lambda \backslash I)^{*}$ be the largest element of $\Lambda$ not in $I$; then, $v_{n}$ has an edge with every vertex $v_{k}$, where $k \in(\Lambda \backslash I)^{*}$ and $k \neq n$. Therefore, $d\left(v_{n}, v_{k}\right)=1$. Now, for any two vertices $v_{i}$ and $v_{j}, i \neq j$, we have

$$
d\left(v_{i}, v_{j}\right) \leq d\left(v_{i}, v_{n}\right)+d\left(v_{n}, v_{j}\right) \leq 2 .
$$

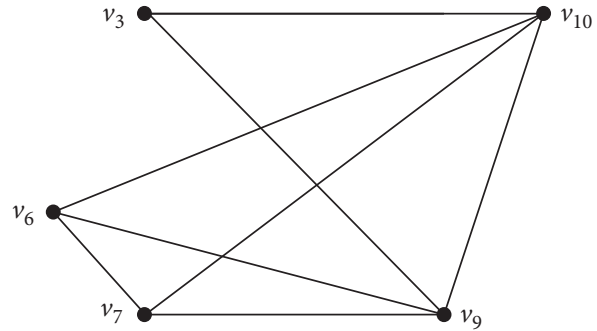

FIgUre 1: Graph $G_{I}(\Lambda)$, where $\Lambda=<3,5,7>$ and $I=\{11,12,13, \ldots\}$.

This implies that

$$
\operatorname{diam}\left(G_{I}(\Lambda)\right) \leq 2 .
$$

Moreover, if any undirected graph $G$ has a cycle, then gr $(G) \leq 2 \operatorname{diam}(G)+1$ (see [13], Propostion 1.3.2). Therefore, $\operatorname{gr}\left(G_{I}(\Lambda)\right) \leq 5$.

Lemma 1. Let $G_{I}(\Lambda)$ be a graph associated with an ideal I of a numerical semigroup $\Lambda$. If order of $G_{I}(\Lambda)$ is 4 , then $G_{I}(\Lambda)$ must contain a cycle of length 3 .

Proof. We may assume that $(\Lambda \backslash I)^{*}=\left\{x_{1}, x_{2}, x_{3}, x_{4}\right\}$. If $G_{I}(\Lambda)$ is complete, then trivially it contains a cycle of length 3 ; otherwise, there is at least one pair of nonadjacent vertices. We have the following cases:

Case_1: if $v_{x_{1}}$ and $v_{x_{2}}$ are nonadjacent, then either $x_{1}+$ $x_{2}=x_{3}$ or $x_{1}+x_{2}=x_{4}$. If $x_{1}+x_{2}=x_{3}$, then there are two more possibilities, either $x_{1}+x_{3}=x_{4}$ or $x_{2}+x_{3}=x_{4}$. So, $x_{1}+x_{2}=x_{3}$ and $x_{1}+x_{3}=x_{4}$ give $x_{2}+x_{3}, x_{2}+x_{4}$, and $x_{3}+x_{4} \in I$. This implies that there is a cycle $v_{x_{2}}-v_{x_{3}}-v_{x_{4}}-v_{x_{2}}$ of length 3 . Also, $x_{1}+x_{2}=x_{3}$ and $x_{2}+x_{3}=x_{4}$ give $x_{1}+x_{3}$, $x_{1}+x_{4}$, and $x_{3}+x_{4} \in I$. This implies there is a cycle $v_{x_{1}}-v_{x_{3}}-v_{x_{4}}-v_{x_{1}}$ of length 3 . Now, if $x_{1}+x_{2}=x_{4}$, then clearly $x_{1}+x_{3}, x_{1}+x_{4}$, and $x_{3}+x_{4} \in I$, and therefore, there is a cycle $v_{x_{1}}-v_{x_{3}}-v_{x_{4}}-v_{x_{1}}$ of length 3 .

Case_2: if $x_{1}$ and $x_{3}$ are nonadjacent, then $x_{2}+x_{3}, x_{2}+x_{4}$, and $x_{3}+x_{4} \in I$, and therefore, there is a cycle $v_{x_{2}}-v_{x_{3}}-v_{x_{4}}-v_{x_{2}}$ of length 3 .

Case_3: if $x_{2}$ and $x_{3}$ are nonadjacent, then $x_{1}+x_{3}, x_{1}+x_{4}$, and $x_{3}+x_{4} \in I$, and therefore, there is a cycle $v_{x_{1}}-v_{x_{3}}-v_{x_{4}}-v_{x_{1}}$ of length 3 .

Proposition 3. Let $G_{I}(\Lambda)$ be a graph associated with an ideal I of a numerical semigroup $\Lambda$. If order of $G_{I}(\Lambda) \geq 4$, then $G_{I}(\Lambda)$ must contain a cycle of length 3 .

Proof. This follows from Lemma 1.

Corollary 1. Let $G_{I}(\Lambda)$ be a graph associated with an ideal I of a numerical semigroup $\Lambda$. If order of $G_{I}(\Lambda) \geq 4$, then $G_{I}(\Lambda)$ is not a bipartite graph. 
Corollary 2. Let $G_{I}(\Lambda)$ be a graph associated with an ideal I of a numerical semigroup $\Lambda$. If order of $G_{I}(\Lambda) \geq 4$, then $\operatorname{gr}\left(G_{I}(\Lambda)\right)=3$.

\section{Completeness of $G_{I}(\Lambda)$}

In this section, we provide a sufficient and necessary condition for $G_{I}(\Lambda)$ to be complete. Moreover, for a given numerical semigroup $\Lambda$, we computed the least number of ideals $I$ for which graph $G_{I}(\Lambda)$ is complete.

Lemma 2. Any ideal of a numerical semigroup $\Lambda$ is an intersection of irreducible ideals. Moreover, every irreducible ideal of numerical semigroup $\Lambda$ is of the form $\Lambda \backslash B(x)$, where $B(x)=\{y \in \Lambda: x-y \in \Lambda\}$, for some $x \in \Lambda$.

Proof. For a proof, see [23].

Proposition 4. Let I be an irreducible ideal of a numerical semigroup $\Lambda$. If order of $G_{I}(\Lambda) \geq 3$, then $G_{I}(\Lambda)$ cannot be a complete graph.

Proof. Since $I$ is an irreducible ideal, order of $G_{I}(\Lambda)=\left|B(x)^{*}\right|$ for some $x \in \Lambda$. We may assume that $B(x)^{*}=\left\{x_{1}, \ldots, x_{n}, x\right\}$, where $n \geq 2$. Then, there must exist $x_{i}, x_{j} \in B(x)^{*}$ for $i \neq j$ such that $x-x_{i}=x_{j}$. This gives $x_{i}+x_{j}=x$, so there is no edge between $v_{x_{i}}$ and $v_{x_{j}}$, and therefore, $G_{I}(\Lambda)$ is not a complete graph.

Example 2. Let $\Lambda=\langle 3,5,7\rangle$ be a numerical semigroup. Choose $10 \in \Lambda$; then, $B^{*}(10)=\{3,5,7,10\}$, and therefore, $I=\Lambda \backslash B^{*}(10)=\{6,8,9,11, \ldots\}$ is an irreducible ideal. As $\left|B^{*}(10)\right|=4, G_{I}(\Lambda)$ is not a complete graph (see Figure 2 ).

Corollary 3. Let $G_{I}(\Lambda)$ is a graph of order $\geq 3$, associated with an irreducible ideal I of a numerical semigroup $\Lambda$. Then, $G_{I}(\Lambda)$ cannot be a regular graph.

Proof. This follows from Proposition 1 and Proposition 4.

Lemma 3. Let $\Lambda=\langle A\rangle$, where $A=\left\{a_{1}, \ldots, a_{r}\right\}$ is the minimal system of generators. Then, for some $x \in \Lambda, B(x)=$ $\left\{0, a_{i}, 2 a_{i}, \ldots, x\right\}$ if and only if $x$ is the multiple of exactly one element $a_{i} \in A$.

Proof. Suppose that $B(x)=\left\{0, a_{i}, 2 a_{i}, \ldots, x\right\}$ and $x$ is not a multiple of exactly one element of $A$. Then,

Case_1: if $x$ is a multiple of an element of $a_{j} \in A$ other than $a_{i}$, then we can assume $x=n a_{j}$. This gives $x-a_{j}=$ $(n-1) a_{j} \in \Lambda$ and therefore $a_{j} \in B(x)$, which is not possible.

Case _2: if $x$ is a linear combination of at least two elements $a_{i}, a_{j} \in A$, then $x=s a_{i}+r a_{j}$ for some positive integers $s$ and $r$. Note that $x-a_{j}=s a_{i}+(r-1) a_{j} \in \Lambda$. This gives $a_{j} \in B(x)$, which is not possible.

The converse of this statement is straightforward.

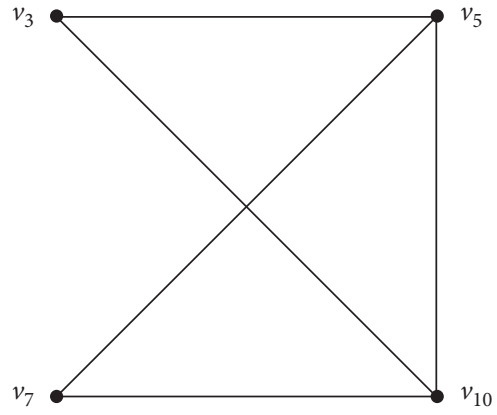

Figure 2: Graph $G_{I}(\Lambda)$, where $\Lambda=<3,5,7>$ and $I$ is an irreducible ideal with $x=10$.

Theorem 1. Let I be an ideal of a numerical semigroup $\Lambda$ such that $I=\cap_{1 \leq j \leq n} I_{j}$, where $I_{j}$ is an irreducible ideal and $n \geq 2$. Then, $G_{I}(\Lambda)$ is complete if and only if order of $G_{I_{j}}(\Lambda) \leq 2$ for each $1 \leq j \leq n$.

Proof. Let $\Lambda=\langle A\rangle$, where $A=\left\{a_{1}, \ldots, a_{r}\right\}$ is the minimal system of generators. As for each $1 \leq j \leq n, I_{j}$ is an irreducible ideal; therefore, we can assume that $I_{j}=\Lambda \backslash B\left(x_{j}\right)$ for some $x_{j} \in \Lambda$. This gives that the set of vertices of $G_{I}(\Lambda)$ is $\left\{v_{i}: i \in B\left(x_{1}\right)^{*} \cup \ldots \cup B\left(x_{n}\right)^{*}\right\}$.

To prove the implication " $\Rightarrow$ ", suppose order of $G_{I_{m}}(\Lambda) \geq 3$ for some $1 \leq m \leq n$. Then, from Proposition 4 , it follows that $G_{I_{m}}(\Lambda)$ is not a complete graph, so there must exist $x_{i}, x_{j} \in B\left(x_{m}\right)^{*}$ such that $x_{i}+x_{j} \in B\left(x_{m}\right)^{*}$; i.e., there is no edge between $v_{x_{i}}$ and $v_{x_{j}}$. This gives $x_{i}, x_{j} \in$ $B\left(x_{1}\right)^{*} \cup \cdots \cup B\left(x_{n}\right)^{*}$ such that $x_{i}+x_{j} \in B\left(x_{1}\right)^{*} \cup$ $\cdots \cup B\left(x_{n}\right)^{*}$, and therefore, $G_{I}(\Lambda)$ is not complete.

Now, to prove the implication " $\models$ ", we suppose on contrary that $G_{I}(\Lambda)$ is not a complete graph. Therefore, there exist $x, y, z \in B\left(x_{1}\right)^{*} \cup \cdots \cup B\left(x_{n}\right)^{*}$ such that $z=x+y$. However, the order of $G_{I_{j}}(\Lambda) \leq 2$ for each $1 \leq j \leq n$; therefore, either $\left|B\left(x_{j}\right)^{*}\right|=1$ or $\left|B\left(x_{j}\right)^{*}\right|=2$. This gives that every element of $B\left(x_{1}\right)^{*} \cup \cdots \cup B\left(x_{n}\right)^{*}$ is either an element of the minimal system of generators $A$ or twice of some element of $A$ (see Lemma 3). Note that $z$ cannot be an element of $A$ because $z=x+y$, so the only possibility is that $z$ is twice of some element of $A$, say $z=2 a_{s}$, for some $1 \leq s \leq r$. This gives $x, y, z \in B(z)^{*}$, i.e., $\left|B(z)^{*}\right| \geq 3$, which is not possible. Hence, $G_{I}(\Lambda)$ is a complete graph.

Example 3. Let $\Lambda=\langle 3,5\rangle=\{0,3,5,6,8,9, \ldots\}$ be a numerical semigroup. Consider the irreducible ideals $I_{1}=\Lambda \backslash B(3)^{*}, I_{2}=\Lambda \backslash B(5)^{*}, I_{3}=\Lambda \backslash B(6)^{*}, I_{4}=\Lambda \backslash B(10)^{*}$, and $I_{5}=\Lambda \backslash B(12)^{*}$, where $B(3)^{*}=\{3\}, B(5)^{*}=\{5\}$, $B(6)^{*}=\{3,6\}, B(10)^{*}=\{5,10\}$, and $B(12)^{*}=\{3,6,9,12\}$. Let $\quad I=I_{1} \cap I_{2} \cap I_{3} \cap I_{4}=\{8,9,11, \ldots\} \quad$ and $J=I_{1} \cap I_{2} \cap I_{3} \cap I_{4} \cap I_{5}=\{11,12, \ldots\}$ be two ideals of $\Lambda$. Then, by Theorem 1, it follows that $G_{I}(\Lambda)$ must be a complete graph (see Figure 3) while $G_{J}(\Lambda)$ cannot be a complete graph (see Figure 4).

Proposition 5. Let $\Lambda=\left\langle a_{1}, \ldots, a_{n}\right\rangle$, where $n \geq 3$ be a $n u$ merical semigroup. Then, there are at least $2^{n}+2^{n-1}$ ideals I such that $G_{I}(\Lambda)$ is complete. 


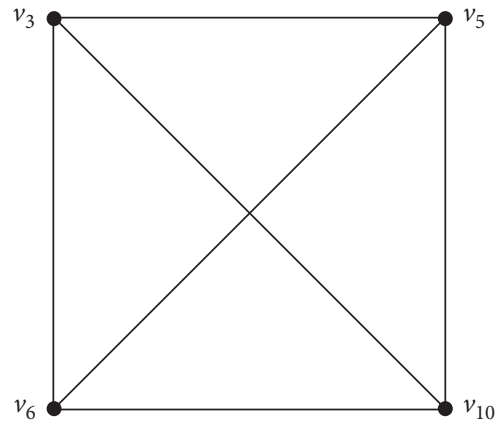

FIGURE 3: Graph $G_{I}(\Lambda)$, where $\Lambda=<3,5,>$ and $I=\{8,9,11, \ldots\}$.

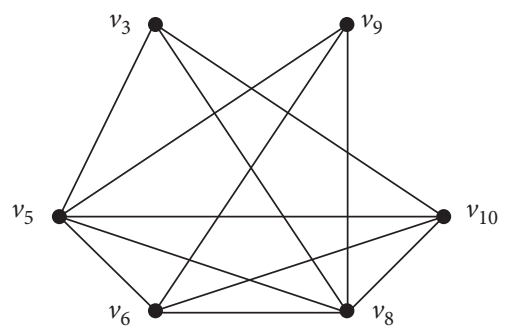

Figure 4: Graph $G_{I}(\Lambda)$, where $\Lambda=<3,5$, $>$ and $I=\{11,12, \ldots\}$.

Proof. We prove it by induction on $n$. If $n=3$, then $\Lambda=\left\langle a_{1}, a_{2}, a_{3}\right\rangle$. We have the following cases:

Case_1: if $I$ is irreducible, then the order of $G_{I}(\Lambda)$ is $\left|B^{*}(x)\right|$. From Theorem 1 , it follows that $G_{I}(\Lambda)$ is complete if and only if $\left|G_{I}(\Lambda)\right| \leq 2$. This gives $\left|B^{*}(x)\right|=1$ or 2 . If $\left|B^{*}(x)\right|=1$, then $B^{*}(x)=\left\{a_{1}\right\},\left\{a_{2}\right\}$, or $\left\{a_{3}\right\}$, and if $\left|B^{*}(x)\right|=2$, then $B^{*}(x)=\left\{a_{1}, 2 a_{1}\right\},\left\{a_{2}, 2 a_{2}\right\}$, or $\left\{a_{3}, 2 a_{3}\right\}$. But, note that $B^{*}(x)=\left\{a_{2}, 2 a_{2}\right\}$ and $\left\{a_{3}, 2 a_{3}\right\}$ may or may not exist because there is a possibility that $2 a_{2}$ and $2 a_{3}$ may be the multiple of more than one element of $\Lambda$ or these are linear combination of two or more than two elements of $\Lambda$. So, in this case, there are at least 4 irreducible ideals $I$ for which $G_{I}(\Lambda)$ is complete.

Case_2: now, if $I$ is not irreducible, then $I$ can be written as an intersection of irreducible ideals. We assume $I=\bigcap_{1 \leq j \leq n} I_{j}$, where $I_{j}$ is an irreducible ideal and $n \geq 2$. This gives order of $G_{I}(\Lambda)$ as $\left|B^{*}(x)=\cup B^{*}\left(x_{j}\right)\right|$. So, if $G_{I}(\Lambda)$ is complete, then $\left|B^{*}\left(x_{j}\right)\right| \leq 2$, for all $j$, and therefore, $B^{*}\left(x_{j}\right)$ is one of $\left\{a_{1}\right\},\left\{a_{2}\right\},\left\{a_{3}\right\},\left\{a_{1}, 2 a_{1}\right\}$, $\left\{a_{2}, 2 a_{2}\right\}$, and $\left\{a_{3}, 2 a_{3}\right\}$. As $\left\{a_{1}\right\},\left\{a_{2}\right\},\left\{a_{3}\right\}$, and $\left\{a_{1}, 2 a_{1}\right\}$ must exist in irreducible case, the least possibilities for $B^{*}(x)$ are $\left\{a_{1}, a_{2}\right\}, \quad\left\{a_{1}, a_{3}\right\}$, $\left\{a_{2}, a_{3}\right\},\left\{a_{1}, a_{2}, 2 a_{1}\right\},\left\{a_{1}, a_{3}, 2 a_{1}\right\},\left\{a_{1}, a_{2}, a_{3}\right\}$, and $\left\{a_{1}, a_{2}, a_{3}, 2 a_{1}\right\}$. This gives that there are at least 7 reducible ideals for which $G_{I}(\Lambda)$ is complete. Moreover, if $\Lambda=I$, then $G_{I}(\Lambda)$ is an empty graph, which is trivially a complete graph. Hence, there are at least $12=$ $2^{3}+2^{2}$ ideals for which $G_{I}(\Lambda)$ is complete.

Now, suppose that, as the induction hypothesis, $n \geq 4$ and, whenever, a numerical semigroup, say $\Lambda_{1}$, has $\left\{a_{1}, a_{2}, \ldots, a_{n-1}\right\}$ as a minimal generating set, then there are at least $2^{n-1}+2^{n-2}$ ideals $J$ such that $G_{J}\left(\Lambda_{1}\right)$ is complete.

Now, let $\Lambda=\left\langle a_{1}, a_{2}, \ldots, a_{n}\right\rangle$. Note that $\left\{a_{1}, a_{2}, \ldots, a_{n}\right\}=\left\{a_{1}, a_{2}, \ldots, a_{n-1}\right\} \cup\left\{a_{n}\right\}$. Then, induction hypothesis gives that there are at least $2\left(2^{n-1}+2^{n-2}\right)=2^{n}+$ $2^{n-1}$ ideals $I$ such that $G_{I}(\Lambda)$ is complete.

\section{Clique Number and Cut Point}

In this section, we present some results on clique number and cut point of the graph $G_{I}(\Lambda)$.

Proposition 6. Let $G_{I}(\Lambda)$ be a graph associated with an irreducible ideal I of a numerical semigroup $\Lambda$. If order of $G_{I}(\Lambda)$ is $n$, then

$$
c l\left(G_{I}(\Lambda)\right)= \begin{cases}\frac{n}{2}+1, & \text { if } n \text { is even, } \\ \frac{n+1}{2}, & \text { if } n \text { is odd }\end{cases}
$$

Proof. Since $I$ is irreducible, the order of $G_{I}(\Lambda)$ is exactly $\left|B(x)^{*}\right|$, for some $x \in \Lambda$. Assume that $B(x)^{*}=\left\{x_{1}, x_{2}, \ldots, x_{n-1}, x\right\}$. We have the following cases:

Case_1: if $\left|B(x)^{*}\right|$ is even, then the cardinality of the set $B(x)^{*}-\{x\}$ is odd, and by definition of $B(x)$, we know that $x-x_{1}=x_{n-1}, x-x_{2}=x_{n-2}, \ldots, \quad x-x_{(n / 2)-1}=$ $x_{(n / 2)+1}$. This gives that $\left(v_{x_{1}}, v_{x_{n-1}}\right),\left(v_{x_{2}}, v_{x_{n-2}}\right), \ldots$, $\left(v_{x_{(n / 2)-1}}, v_{x_{(n / 2)+1}}\right)$ are pairs of nonadjacent vertices. As cardinality of $B(x)^{*}-\{x\}$ is odd, it must contain an element $x_{(n / 2)}=x / 2$. Note that every element of the set $\left\{x_{(n / 2)}, x_{(n / 2)+1}, \ldots, x_{n-1}, x\right\}$ is equal or greater than $x / 2$, and therefore, the sum of any two different elements is greater than $x$. This gives that the graph corresponding to the set $\left\{x_{(n / 2)}, x_{(n / 2)+1}, \ldots, x_{n-1}, x\right\}$ is a complete graph of maximum possible order.

Case_2: if $\left|B(x)^{*}\right|$ is odd, then the cardinality of the set $B(x)^{*}-\{x\}$ is even. In this case, we have $x-x_{1}=x_{n-1}, x-x_{2}=x_{n-2}, \ldots, x-x_{(n-1) / 2}=x_{(n+1) / 2}$. This gives that $\left(v_{x_{1}}, v_{x_{n-1}}\right),\left(v_{x_{2}}, v_{x_{n-2}}\right), \ldots$, $\left(v_{x_{(n-1) / 2}}, v_{x_{(n+1) / 2}}\right)$ are pairs of nonadjacent vertices. Note that every element of the set $\left\{x_{(n+1) / 2}, x_{((n+1) / 2)+1}, \ldots, x_{n-1}, x\right\}$ is greater than $x / 2$, and therefore, the sum of any two different elements is greater than $x$. This gives that the graph corresponding to the set $\left\{x_{((n+1) / 2)}, x_{\left.((n+t 1) / 2 n+q 1), \ldots, x_{n-1}, x\right\}}\right.$ is a complete graph of maximum possible order.

Example 4. Let $\Lambda=\langle 3,5\rangle$ be a numerical semigroup and $I=\Lambda \backslash B^{*}(16)=\{9,12,14,15,17, \ldots\}$ be an irreducible ideal of $\Lambda$. Then, Proposition 6 gives that the subset $\{8,10,11,13,16\}$ of $B^{*}(16)=\{3,5,6,8,10,11,13,16\}$ is the maximal clique, and therefore, the clique number of graph 


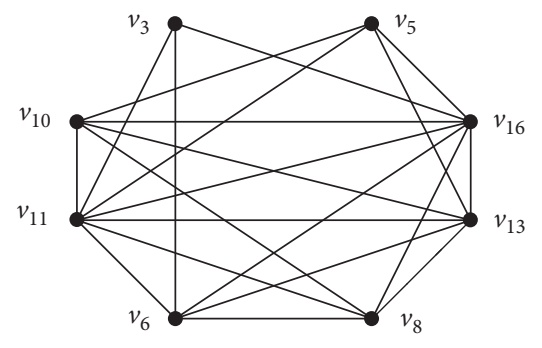

FIGURE 5: Graph $G_{I}(\Lambda)$, where $\Lambda=<3,5$, $>$ and $I$ is an irreducible ideal with $x=16$.

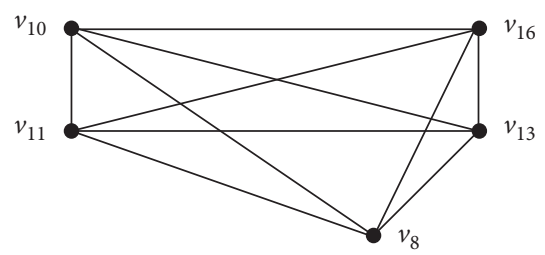

Figure 6: Clique of graph $G_{I}(\Lambda)$ given in Figure 5.

$G_{I}(\Lambda)$ is 5 . The graph $G_{I}(\Lambda)$ and its corresponding maximal clique are given in Figures 5 and 6, respectively:

Proposition 7. Let $\Lambda=\langle A\rangle$ be a numerical semigroup, where $A=\left\{a_{1}, a_{2}, \ldots, a_{n}\right\}$ is the minimal system of generators of $\Lambda$ and $G_{I}(\Lambda)$ is a graph associated with an irreducible ideal $I=\Lambda \backslash B^{*}(x)$, for some $x \in \Lambda$ such that the order of $G_{I}(\Lambda) \geq 3$. Then, $v_{x}$ is the only cut point of $G_{I}(\Lambda)$ if and only if $B^{*}(x)=\left\{a_{i}, 2 a_{i}, \ldots, x\right\}$ for some $a_{i} \in A$.

Proof. Assume that there exist some $y_{1}, \ldots, y_{k} \in B^{*}(x)$ such that $y_{j} \neq n a_{i}$, where $1 \leq n \leq m$ and $1 \leq j \leq k$. Then, we have the following cases:

Case_1: if $j=1$, then clearly $y_{1}+n a_{i} \in I$ for all $1 \leq n \leq m$; otherwise, $y_{1}$ is a multiple of $a_{i}$. This gives that for every two elements $x_{1}, x_{2} \in B^{*}(x)$ other than $x$ and $y_{1}$, we have two different paths $v_{x_{1}}-v_{x}-v_{x_{2}}$ and $v_{x_{1}}-v_{y_{1}}-v_{x_{2}}$. This implies that $v_{x}$ is not a cut point of $G_{I}(\Lambda)$.

Case_2: if $j>1$, then assume that $y_{k} \in B^{*}(x)$ is the largest element such that $y_{k} \neq n a_{i}$, where $1 \leq n \leq m$. Note that $y_{k}+y_{1}=x$, where $y_{1}$ is the smallest element of $B^{*}(x)$ such that $y_{1} \neq n a_{i}$ and $1 \leq n \leq m$. Then, $y+y_{k} \in I$, for all $y \in B^{*}(x)-\left\{y_{1}\right\} \quad$ and $y_{1}+(m-1) a_{i} \in I$. This gives that for every two elements $x_{1}, x_{2} \in B^{*}(x)$ other than $x$ and $y_{k}$, we have two different paths, either $v_{x_{1}}-v_{x}-v_{x_{2}}$ and $v_{x_{1}}-v_{y_{k}}-v_{x_{2}}$ or $v_{x_{1}}-v_{x}-v_{x_{2}}$ and $v_{x_{1}}-v_{(m-1) a_{i}}-v_{y_{k}}-v_{x_{2}}$. This implies that $v_{x}$ is not a cut point of $G_{I}(\Lambda)$.

Now, if $B^{*}(x)=\left\{a_{i}, 2 a_{i}, \ldots, x\right\}$, then clearly $a_{i}+k a_{i} \in B^{*}(x) \forall i \leq k \leq m-1$ and $a_{i}+x \in I$. This gives that $G_{I}(\Lambda)-\left\{v_{x}\right\}$ has at least two disconnected components and $y+x \in I$ for all $y \in B^{*}(x)-\{x\}$. Therefore, $x$ is the only cut point.

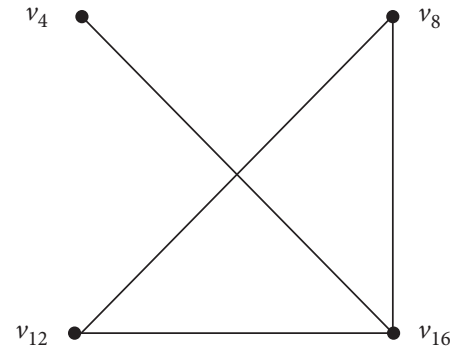

FIgURE 7: Graph $G_{I}(\Lambda)$, where $\Lambda=<4,5$, $>$ and $I$ is an irreducible ideal with $x=16$.

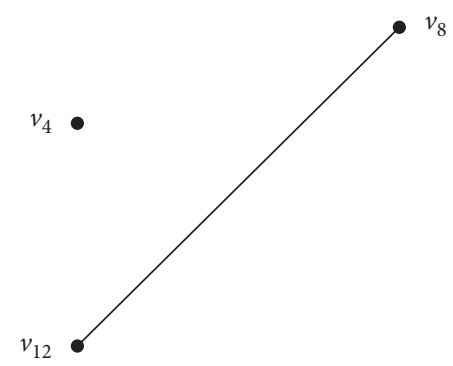

Figure 8: Graph $G_{I}(\Lambda)-\left\{v_{16}\right\}$.

Example 5. Let $\Lambda=\langle 4,5$,$\rangle be a numerical semigroup and$ $I=\Lambda \backslash B^{*}(16)$ be a irreducible ideal, where $B^{*}(16)=$ $\{4,8,12,16\}$. Then, $v_{16}$ is a cut point of graph $G_{I}(\Lambda)$ (see Figures 7 and 8 ).

\section{Conclusion}

In this article, graphs associated with ideals of a numerical semigroup have been studied and it has been proved that these graphs are connected. We investigated some properties such as girth, regularity, clique number, and cut point of these graphs. Also, a necessary and sufficient condition has been given for a graph associated with an ideal of a numerical semigroup to be complete.

\section{Data Availability}

No data were used to support this study.

\section{Disclosure}

This research was carried out as a part of the employment of the authors.

\section{Conflicts of Interest}

The authors declare that there are no conflicts of interest regarding the publication of this paper.

\section{References}

[1] A. T. Balaban, "Applications of graph theory in chemistry," Journal of Chemical Information and Modeling, vol. 25, no. 3, pp. 334-343, 1985. 
[2] O. Mason and M. Verwoerd, "Graph theory and networks in biology," IET Systems Biology, vol. 1, no. 2, pp. 89-119, 2007.

[3] S. G. Shirinivas, S. Vetrivel, and N. M. Elango, "Applications of graph theory in computer science an overview," International Journal of Engineering Science and Technology, vol. 2, no. 9, pp. 4610-4621, 2010.

[4] M. Afkhami and K. Khashyarmanesh, "The intersection graph of ideals of a lattice, note," Mat, vol. 34, no. 2, pp. 135-143, 2014.

[5] S. Akbari, H. A. Tavallaee, and S. K. Ghezelahmad, "Intersection graph of submodules of a module," Journal of Algebra and Its Applications, vol. 11, no. 1, pp. 1-8, Article ID 1250019, 2012.

[6] D. F. Anderson and A. Badawi, "The total graph of a commutative ring," Journal of Algebra, vol. 320, no. 7, pp. 2706-2719, 2008.

[7] I. Beck, "Coloring of commutative rings," Journal of Algebra, vol. 116, no. 1, pp. 208-226, 1998.

[8] S. Ebrahimi Atani, S. Dolati, M. Khoramdel, and M. Sedghi, "Total graph of a 0-distributive lattice," Categories and General Algebraic Structures with Applications, vol. 9, no. 1, pp. 15-27, 2018.

[9] E. Hashemi, A. Alhevaz, and E. Yoonesian, "On zero divisor graph of unique product monoid rings over Noetherian reversible ring," Categories and general algebraic structures with applications, vol. 4, no. 1, pp. 95-113, 2016.

[10] R. Shen, "Intersection graphs of subgroups of finite groups," Czechoslovak Mathematical Journal, vol. 60, no. 4, pp. 945950, 2010.

[11] E. Yaraneri, "Intersection graph of a module," Journal of Algebra and Its Applications, vol. 12, no. 5, pp. 1-30, 2013.

[12] G. Chartrand, Introduction to Graph Theory, Tata McGrawHill Education, Bengaluru, Karnataka, 2006.

[13] R. Diestel, Graph Theory, Springer-Verlag, New York, NY, USA, 1997.

[14] D. B. West, Introduction to Graph Theory, Vol. 2, PrenticeHall, Upper Saddle River, NY, USA, 2001.

[15] H. Greenberg, "Solution to a linear diophantine equation for nonnegative integers," Journal of Algorithms, vol. 9, no. 3, pp. 343-353, 1988.

[16] O. J. Rodseth, "Linear diophantine problem of Frobenius," Journal fur die reine und angewandte Mathematik, vol. 307, pp. 431-440, 1979.

[17] E. S. Selmer, "On a linear diophantine problem of Frobenius," Journal für die reine und angewandte Mathematik, vol. 293/ 294, pp. 1-17, 1997.

[18] J. J. Sylvester, "Mathematical questions with their solutions," Educational Times, vol. 41, p. 21, 1884.

[19] G. L. Feng and T. R. N. Rao, "A simple approach for construction of algebraic-geometric codes from affine plane curves," IEEE Transactions on Information Theory, vol. 40, no. 4, pp. 1003-1112, 1994.

[20] T. HØholdt, J. H. van Lint, and R. Pellikaan, "Algebraic geometry codes," in Handbook of Coding Theory, V. S. Pless, W. C. Huffman, and R. A. Brauldi, Eds., vol. I, pp. 871-961, Elsevier, Amsterdam, Netherlands, 1998.

[21] C. Kirfel and R. Pellikaan, "The minimum distance of codes in an array coming from telescopic semigroups," IEEE Transactions on Information Theory, vol. 41, no. 6, pp. 1720-1732, 1995.

[22] M. A. Binyamin, H. M. A. Siddiqui, N. M. Khan, A. Aslam, and Y. Rao, "Characterization of graphs associated with numerical semigroups," Mathematics, vol. 7, no. 6, p. 557, 2019.
[23] V. Barucci, "Decompositions of ideals into irreducible ideals in numerical semigroups," Journal of Commutative Algebra, vol. 2, no. 3, pp. 281-294, 2010. 